\title{
APPLICATIONS OF MOMENT PROBLEMS TO THE OVERCOMPLETENESS OF SEQUENCES
}

\author{
ISABELLE CHALENDAR and JONATHAN R. PARTINGTON
}

\begin{abstract}
This paper introduces the notion of a determining function, which is used in order to apply results on the determination of measures by moments to the theory of overcompleteness of sequences in various function spaces, providing strong generalizations of results of Lin and Too. A further application is given to the determination of probability distributions by means of moments of record values.
\end{abstract}

\section{Introduction}

Problems involving the determination of a measure from its moments are of importance in many areas of analysis and probability. (See, for example, [8].) Recently, there has been some work to the effect that under some circumstances the moments of two measures need not be assumed to be equal, since as soon as they are approximately equal (in a sense to be made precise later), this forces the measures to differ on a small set; stronger forms of such results force the measures even to coincide [2], [3], [9], [12]. Such results can be applied in the geometry of Banach spaces and in areas of probability theory. In order to derive some of these as clearly as possible, we shall introduce the key notion of a determining function, which is, roughly speaking, a function $\Phi$ such that the measure $\Phi(x) d x$ is uniquely determined by its moments.

The main application that we have in mind is towards the overcompleteness of sequences in Hardy and Bergman spaces. Recall that a sequence $\left(x_{m}\right)_{m}$ in a Banach space $\mathscr{X}$ is said to be complete if its closed linear span is the whole space $\mathscr{X}$, and overcomplete (or sometimes hypercomplete or densely closed) if every infinite subsequence of $\left(x_{m}\right)_{m}$ is complete in $\mathscr{X}$ (see, for example, the book of Singer [13], and the very recent article [1]).

A weaker but more useful notion is finite overcompleteness: a sequence is finitely overcomplete, when it remains complete on deleting finitely many terms from the sequence. For example, the functions $\left(f_{m}\right)_{m}$ with $f_{m}(x)=x^{m}$ for $x \in(0,1)$ can be shown to form a finitely overcomplete sequence in $L^{2}(0,1)$

Received February 27, 2006. 
by means of the Stone-Weierstrass theorem. Finitely overcomplete sequences have many applications in signal-processing, for example via the theory of frames [7].

Thus, as an application of the theory of moments, we shall provide extensive generalizations of Lin's result [10] that the sequence of functions $\left(x^{m} e^{-\lambda x}\right)_{m}$ is finitely overcomplete in $L^{1}(0, \infty)$ for any $\lambda>0$. Here we work with weighted Lebesgue spaces, Hardy spaces and Bergman spaces, and in addition we give some multivariable extensions.

We adopt the following conventions. Let $\mathbf{N}=\{0,1,2, \ldots\}, \mathbf{R}_{+}=\{x \in \mathbf{R}$ : $x \geq 0\}$ and similarly for $\mathrm{N}^{n}$ and $\mathrm{R}_{+}^{n}$. Also $\mathrm{C}_{+}=\{z \in \mathrm{C}: \operatorname{Re} z>0\}$.

For a given vector $x=\left(x_{1}, \ldots, x_{n}\right) \in \mathbf{R}^{n}$ and a multi-index $\alpha=\left(\alpha_{1}, \ldots\right.$, $\left.\alpha_{n}\right) \in \mathbf{N}^{n}$, we write $x^{\alpha}$ to denote $x_{1}^{\alpha_{1}} \ldots x_{n}^{\alpha_{n}}$.

A function $f: \mathrm{R}_{+}^{n} \rightarrow \mathrm{C}$ is said to be of polynomial growth, if there exist constants $C, d>0$ such that

$$
|f(x)| \leq C\left(1+\|x\|^{d}\right), \quad\left(x \in \mathrm{R}_{+}^{n}\right) .
$$

Similarly for functions defined on $\mathrm{R}^{n}$.

The symbol $\delta_{x_{0}}$ denotes a Dirac mass (atomic probability measure) supported on $\left\{x_{0}\right\}$.

The structure of this paper is as follows. In Section 2 we introduce the notion of a determining function, which enables us to present various results on the characterization of finite complex measures on $\mathrm{R}_{+}^{n}$ or $\mathrm{R}^{n}$ by means of their moments. The main application of this is provided in Section 3, where we explicitly construct a large class of finitely overcomplete sequences (sequences that remain complete even after the removal of finitely-many terms) in weighted Lebesgue spaces and also Hardy and Bergman spaces on $\mathrm{C}_{+}$; in the latter case the sequences can be seen as perturbations of a function and its derivatives. A further application is presented in Section 4, to the determination of probability distributions by means of the moments of record values, extending results in [12].

\section{Approximate Carleman theorems}

Definition 2.1. A function $\Phi: \mathbf{R}_{+} \rightarrow \mathbf{R}$ is said to be a determining function, if $\Phi$ is Borel measurable with $\Phi(x) \neq 0$ for $x \neq 0$, and $\Phi$ has the property that for each $m \geq 0$ the function $\psi_{m}$ given by $\psi_{m}(x)=x^{m} \Phi(x)$ is bounded and

$$
\sum_{m=0}^{\infty}\left\|\psi_{m}\right\|_{\infty}^{-1 /(2 m)}=\infty .
$$


Likewise, a function $\Phi: \mathbf{R} \rightarrow \mathbf{R}$ is said to be a determining function, if $\Phi$ is Borel measurable with $\Phi(x) \neq 0$ for $x \neq 0$, and $\Phi$ has the property that for each $m \geq 0$ the function $\psi_{2 m}$ given by $\psi_{2 m}(x)=x^{2 m} \Phi(x)$ is bounded and

$$
\sum_{m=0}^{\infty}\left\|\psi_{2 m}\right\|_{\infty}^{-1 /(2 m)}=\infty .
$$

For example, on $[0, \infty)$, if $\Phi(x)=e^{-\lambda x^{\delta}}$ where $\lambda>0$ and $\delta \geq \frac{1}{2}$, then $\left\|\psi_{m}\right\|_{\infty}^{-1 /(2 m)} \sim C m^{-1 /(2 \delta)}$, for some constant $C>0$, and if $\Phi(x)=$ $e^{-(\lambda x / \log (x+2))^{1 / 2}}$, then $\left\|\psi_{m}\right\|_{\infty}^{-1 /(2 m)} \sim C(m \log m)^{-1}$.

Similarly, on R, the function $\Phi(x)=e^{-\lambda|x|^{\delta}}$ satisfies $\left\|\psi_{2 m}\right\|_{\infty}^{-1 /(2 m)} \sim$ $C m^{-1 / \delta}$, so $\Phi$ is a determining function provided that $\delta \geq 1$.

Note that, if $\Phi$ is a determining function, then so is the product $\gamma \Phi$, whenever $\gamma$ is a Borel measurable function of polynomial growth such that $\gamma(x) \neq 0$ for $x \neq 0$. This follows because

$$
\left|x^{m} \gamma(x) \Phi(x)\right| \leq C\left(\left\|\psi_{m}\right\|_{\infty}+\left\|\psi_{m+d}\right\|_{\infty}\right),
$$

in the notation of (1).

Lemma 2.2. Let $\mu$ be a finite Borel measure on $\mathbf{R}_{+}$, and $\Phi$ a determining function on $\mathrm{R}_{+}$. Then the moments $M_{m}$, defined by

$$
M_{m}=\int_{0}^{\infty} x^{m}|\Phi(x)| d \mu(x),
$$

satisfy the Carleman condition

$$
\sum_{m=0}^{\infty}\left|M_{m}\right|^{-1 /(2 m)}=\infty .
$$

Similarly, for $\mu$ a finite Borel measure on $\mathrm{R}$, and $\Phi$ a determining function on $\mathrm{R}$, the moments $M_{m}$ defined by

$$
M_{m}=\int_{-\infty}^{\infty}|x|^{m}|\Phi(x)| d \mu(x),
$$

satisfy the Carleman condition

$$
\sum_{m=0}^{\infty}\left|M_{2 m}\right|^{-1 /(2 m)}=\infty .
$$


Proof. We have

$$
\left|M_{m}\right| \leq\|\mu\|\left\|\psi_{m}\right\|_{\infty}
$$

from which the result follows easily.

Lin and Too [12] showed that if $\mu_{1}$ and $\mu_{2}$ are two absolutely continuous probability measures such that

$$
\int_{0}^{\infty} x^{m} e^{-\lambda x} d \mu_{1}(x)=\int_{0}^{\infty} x^{m} e^{-\lambda x} d \mu_{2}(x)+c,
$$

for sufficiently large $m$ and for some constants $c$ and $\lambda>0$, then $\mu_{1}=\mu_{2}$.

This can be put into the wider context of determining functions, and one may state a much more general theorem as follows.

THEOREM 2.3. Let $\Omega=\mathrm{R}$ or $\mathrm{R}_{+}$, and let $\mu_{1}, \mu_{2}$ be two finite complex Borel measures on $\Omega$. Let $\Phi$ be a determining function on $\Omega$. Suppose that there exist constants $c$ and $\alpha$ such that for each $m \geq 0$

$$
\int_{\Omega} x^{m} \Phi(x) d \mu_{1}(x)=\int_{\Omega} x^{m} \Phi(x) d \mu_{2}(x)+c_{m},
$$

where $c_{m}$ satisfies $\lim \sup \left|c_{m}-c\right|^{1 / m}=\alpha$. Then, there exists a complex measure $v$ supported on $[-\alpha, \alpha] \cap \Omega$ such that

$$
\mu_{1}=\mu_{2}+\frac{c}{\Phi(1)} \delta_{1}+v
$$

Proof. Set

$$
d_{m}=\int_{\Omega} x^{m} \Phi(x) d\left(\mu_{1}-\mu_{2}-\frac{c}{\Phi(1)} \delta_{1}\right)(x) .
$$

Then lim sup $\left|d_{m}\right|^{1 / m}=\alpha$. Define a measure $\rho$ by

$$
d \rho(x)=\Phi(x) d\left(\mu_{1}-\mu_{2}-\frac{c}{\Phi(1)} \delta_{1}\right)(x) .
$$

By Lemma 2.2, the numbers $M_{m}=\int_{\Omega}|x|^{m} d|\rho|(x)$ satisfy the Carleman condition (4) or (5), as appropriate. When the measures are signed real Borel measures, it follows from [9, Cor. 2.4] and [2, Thm. 3.1] - the general case is in $[3$, Thm. 3.1] - that $\rho$ is supported on $[-\alpha, \alpha] \cap \Omega$. Since $\Phi$ does not vanish on $((-\infty,-\alpha) \cup(\alpha, \infty)) \cap \Omega$, the result now follows. 
CoROllary 2.4. Let $X$ and $Y$ be two nonnegative random variables. Let $\Phi$ be a determining function on $\mathbf{R}_{+}$. Suppose that there exist constants $c$ and $c_{m}$ $(m \geq 1)$ such that

$$
E\left(X^{m} \Phi(X)\right)=E\left(Y^{m} \Phi(Y)\right)+c_{m},
$$

where $\lim \sup \left|c_{m}-c\right|^{1 / m}=0$. Then $X$ and $Y$ have the same distribution apart from atomic measures of size $\frac{c}{\Phi(1)}$ concentrated at 0 and 1 . In the particular case where the distributions are purely non-atomic, they are the same.

Proof. By Theorem 2.3, the probability measures $\mu_{1}$ and $\mu_{2}$ associated with $X$ and $Y$ satisfy $\mu_{1}=\mu_{2}+\frac{c}{\Phi(1)} \delta_{1}+v$, with $v$ supported by $\{0\}$. Since $\mu_{1}$ and $\mu_{2}$ are probability measures, $v=-\frac{c}{\Phi(1)} \delta_{0}$. The corollary follows.

It is convenient to give multivariable versions of the above results.

TheOREM 2.5. Let $\Omega$ be either $\mathbf{R}$ or $\mathbf{R}_{+}$, let $\mu_{1}$ and $\mu_{2}$ be finite complex Borel measures on $\Omega^{n}$. Let $\tilde{1}:=(1,1, \ldots, 1)$ and $\Phi_{1}, \ldots, \Phi_{n}$ be determining functions on $\Omega$. Set $\Phi(x)=\prod_{k=1}^{n} \Phi_{k}\left(x_{k}\right)$ for $x=\left(x_{1}, \ldots, x_{n}\right) \in \Omega^{n}$. Suppose that

$$
\int_{\Omega^{n}} x^{\alpha} \Phi(x) d \mu_{1}(x)=\int_{\Omega^{n}} x^{\alpha} \Phi(x) d \mu_{2}(x)+c(\alpha),
$$

where $c(\alpha)$ satisfies the condition that for each $\epsilon>0$ there is a number $A_{\epsilon}>0$ such that

$$
|c(\alpha)-c| \leq A_{\epsilon}\left(c_{1}+\epsilon\right)^{\alpha_{1}} \ldots\left(c_{n}+\epsilon\right)^{\alpha_{n}}
$$

for some fixed number $c$ and constants $c_{1}, \ldots, c_{n}$. Then there is a complex measure $v$ supported on $\prod_{k=1}^{n}\left[-c_{k}, c_{k}\right] \cap \Omega^{n}$ such that

$$
\mu_{1}=\mu_{2}+\frac{c}{\Phi(\tilde{1})} \delta_{\tilde{1}}+v .
$$

Proof. Set

$$
d(\alpha)=\int_{\Omega^{n}} x^{\alpha} \Phi(x) d\left(\mu_{1}-\mu_{2}-\frac{c}{\Phi(\tilde{1})} \delta_{\tilde{1}}\right)(x) .
$$

Then for each $\epsilon>0$ we have

$$
|d(\alpha)| \leq A_{\epsilon}\left(c_{1}+\epsilon\right)^{\alpha_{1}} \ldots\left(c_{n}+\epsilon\right)^{\alpha_{n}} .
$$

Define a measure $\rho$ by

$$
d \rho(x)=\Phi(x) d\left(\mu_{1}-\mu_{2}-c \frac{c}{\Phi(\tilde{1})} \delta_{\tilde{1}}\right)(x) .
$$


As in the proof of Theorem 2.3, consider the numbers

$$
M(j, m)=\int_{\Omega}\left|x_{j}\right|^{m} d|\rho|(x) .
$$

Since $\Phi_{j}$ is a determining function, we see, as in the proof of Lemma 2.2, that for each $j$ the sequence $(M(j, m))_{m}$ satisfies the Carleman condition. The result now follows from [3, Thm. 3.1].

The next corollary follows as in the one-dimensional case.

COROLlary 2.6. Given the hypotheses of Theorem 2.5, suppose that

$$
\limsup _{|\alpha| \rightarrow \infty}|c(\alpha)-c|^{1 /|\alpha|}=0 .
$$

Then $\mu_{1}=\mu_{2}+\frac{c}{\Phi(\tilde{1})}\left(\delta_{\tilde{1}}-\delta_{\tilde{0}}\right)$, where $\tilde{0}=(0,0, \ldots, 0)$. In the particular case when $\mu_{1}$ and $\mu_{2}$ are purely non-atomic, then they are equal.

Another straightforward corollary holds for radial weights.

COROLlaRY 2.7. Let $\Omega=\mathbf{R}$ or $\mathbf{R}_{+}$and let $\mu_{1}$ and $\mu_{2}$ be two finite complex measures on $\Omega^{n}$. Let $\Phi$ be a determining function on $\Omega$. Suppose that

$$
\int_{\Omega^{n}} x^{\alpha} \Phi(\|x\|) d \mu_{1}(x)=\int_{\Omega^{n}} x^{\alpha} \Phi(\|x\|) d \mu_{2}(x)+c(\alpha),
$$

where $c(\alpha)$ satisfies the condition that for each $\epsilon>0$ there is a number $A_{\epsilon}>0$ such that

$$
|c(\alpha)-c| \leq A_{\epsilon}\left(c_{1}+\epsilon\right)^{\alpha_{1}} \ldots\left(c_{n}+\epsilon\right)^{\alpha_{n}}
$$

for some fixed number $c$ and constants $c_{1}, \ldots, c_{n}$. Then there is a complex measure $v$ supported on $\prod_{k=1}^{n}\left[-c_{k}, c_{k}\right] \cap \Omega^{n}$ such that

$$
\mu_{1}=\mu_{2}+\frac{c}{\Phi(\sqrt{n})} \delta_{\tilde{1}}+v \text {. }
$$

Proof. The proof follows along standard lines.

\section{Finitely overcomplete sequences}

In this section we derive some applications of moment problems to the theory of Banach spaces.

Definition 3.1. A sequence $\left(x_{m}\right)_{m}$ in a Banach space is finitely overcomplete if it remains complete (that is, its linear span is dense) whenever finitely many terms are removed. 
In [10] Lin showed that the sequence of functions $\left(x^{m} e^{-\lambda x}\right)_{m}$ is finitely overcomplete in $L^{1}\left(\mathrm{R}_{+}\right)$for any $\lambda>0$. Much more can be proved using the method of moments.

Theorem 3.2. Let $\Omega$ be either $\mathbf{R}$ or $\mathbf{R}_{+}$, let $p \in[1, \infty)$, and let $\Phi$ be a determining function on $\Omega$ such that $x \mapsto \Phi(x)^{\epsilon} \in L^{p}(\Omega)$ for some $\epsilon \in(0,1)$. Suppose that the functions $\Psi_{m} \in L^{p}(\Omega)$ satisfy $\lim \left\|\Psi_{m}\right\|_{p}^{1 / m}=0$. Then the sequence $\left(f_{m}\right)_{m}$ given by

$$
f_{m}(x)=x^{m} \Phi(x)+\Psi_{m}(x),
$$

is finitely overcomplete in $L^{p}(\Omega)$.

Proof. Note that

$$
\begin{aligned}
\int_{\Omega}|x|^{m p} \Phi(x)^{p} d x & =\int_{\Omega}|x|^{m p} \Phi(x)^{p(1-\epsilon)} \Phi(x)^{p \epsilon} d x \\
& \leq C_{m} \int_{\Omega} \Phi(x)^{p \epsilon} d x,
\end{aligned}
$$

where $C_{m}:=\sup \left\{|x|^{m p} \Phi(x)^{p(1-\epsilon)}: x \in \Omega\right\}<\infty$, since $\Phi$ is determining. So the functions $f_{m}$ lie in $L^{p}(\Omega)$. Suppose that $g \in L^{q}(\Omega)$ with $q=\frac{p}{p-1}$ and $\|g\|_{q}=1$; if $\int_{\Omega} g(x) f_{m}(x) d x=0$ for $m \geq m_{0}$, then

$$
\left|\int_{\Omega}\left(x^{m} \Phi(x)^{1-\epsilon}\right)\left(g(x) \Phi(x)^{\epsilon}\right) d x\right| \leq\left\|\Psi_{m}\right\|_{p} .
$$

Also $g(x) \Phi(x)^{\epsilon} d x$ is a finite purely non-atomic measure and $x \mapsto \Phi(x)^{1-\epsilon}$ is also a determining function. Hence, by Theorem 2.3, $g$ is zero almost everywhere. By the Hahn-Banach theorem, $\left(f_{m}\right)_{m}$ is finitely overcomplete in $L^{p}(\Omega)$.

On taking some examples of determining functions given after Definition 2.1, the above theorem provides a far-reaching generalization of Lin's result, which we state separately, as follows.

COROLlaRY 3.3. Let $\gamma: \mathbf{R}_{+} \rightarrow \mathbf{C}$ be a function of polynomial growth such that $\gamma(x) \neq 0$ except possibly at 0 , and let $\left(\Psi_{m}\right)$ be an arbitrary sequence in $L^{p}\left(\mathbf{R}_{+}\right)$such that $\lim \left\|\Psi_{m}\right\|_{p}^{1 / m}=0$. Then the functions $f_{m}$ defined by

$$
f_{m}(x)=x^{m} \gamma(x) e^{-\lambda x^{\delta}}+\Psi_{m}(x)
$$

form a finitely overcomplete set in $L^{p}\left(\mathbf{R}_{+}\right)$for every $p$ with $1 \leq p<\infty$, for each $\lambda>0$ and $\delta \geq \frac{1}{2}$. Similarly, the functions $g_{m}$ defined by

$$
g_{m}(x)=x^{m} \gamma(x) e^{-\lambda\left(\frac{x}{\log (x+2)}\right)^{1 / 2}}+\Psi_{m}(x)
$$


form a finitely overcomplete set in $L^{p}\left(\mathbf{R}_{+}\right)$for each $\lambda>0$.

Alternatively, let $\gamma: \mathbf{R} \rightarrow \mathrm{C}$ be function of polynomial growth such that $\gamma(x) \neq 0$ except possibly at 0 , and let $\left(\Psi_{m}\right)$ be an arbitrary sequence in $L^{p}(\mathrm{R})$ such that $\lim \left\|\Psi_{m}\right\|_{p}^{1 / m}=0$. Then the functions $f_{m}$ defined by

$$
f_{m}(x)=x^{m} \gamma(x) e^{-\lambda|x|^{\delta}}+\Psi_{m}(x)
$$

form a finitely overcomplete set in $L^{p}(\mathrm{R})$ for every $p$ with $1 \leq p<\infty$, for each $\lambda>0$ and $\delta \geq 1$.

As before, it is possible to derive multivariable extensions of the above results.

Theorem 3.4. Let $\Omega=\mathbf{R}$ or $\mathbf{R}_{+}$, let $p \in[1, \infty)$ and $\Phi_{1}, \ldots, \Phi_{n}$ be determining functions on $\Omega$. Define $\Phi: \Omega^{n} \rightarrow \mathbf{R}_{+}$by

$$
\Phi(x)=\Phi_{1}\left(x_{1}\right) \cdots \Phi_{n}\left(x_{n}\right) \quad \text { for } \quad x=\left(x_{1}, \ldots, x_{n}\right) \in \Omega^{n},
$$

and suppose that $x \mapsto \Phi(x)^{\epsilon} \in L^{p}\left(\Omega^{n}\right)$ for some $\epsilon \in(0,1)$. Suppose that the functions $\Psi_{\alpha} \in L^{p}\left(\mathrm{R}^{n}\right)$ satisfy $\lim \sup _{|\alpha| \rightarrow \infty}\left\|\Psi_{\alpha}\right\|_{p}^{1 /|\alpha|}=0$. Then the set $\left(f_{\alpha}\right)_{\alpha}$ given by

$$
f_{\alpha}(x)=x^{\alpha} \Phi(x)+\Psi_{\alpha}(x),
$$

is finitely overcomplete in $L^{p}\left(\Omega^{n}\right)$.

Proof. As in the proof of Theorem 3.2, it is easily verified that the given functions lie in $L^{p}$. Once again we use duality, supposing that $g \in L^{q}\left(\Omega^{n}\right)$ satisfies

$$
\int_{\Omega^{n}} g(x) f_{\alpha}(x) d x=0
$$

for all but finitely many $\alpha$. We deduce from Theorem 2.5 that $g=0$ almost everywhere.

This has the following immediate consequence.

CoRollary 3.5. Let $\gamma: \mathbf{R}_{+}^{n} \rightarrow \mathbf{C}$ be a function of polynomial growth such that $\gamma(x) \neq 0$ except possibly at 0 , and let $\left(\Psi_{\alpha}\right)_{\alpha \in \mathrm{N}^{n}}$ be a family of functions in $L^{p}\left(\mathrm{R}_{+}^{n}\right)$ such that lim $\sup _{|\alpha| \rightarrow \infty}\left\|\Psi_{\alpha}\right\|_{p}^{1 /|\alpha|}=0$. Then for every $p$ with $1 \leq p<\infty$, and for all $\lambda_{1}, \ldots, \lambda_{n}>0$ and $\delta_{1}, \ldots, \delta_{n} \geq \frac{1}{2}$, the functions $f_{\alpha}$ defined by

$$
f_{\alpha}(x)=x^{\alpha} \gamma(x) e^{-\left(\lambda_{1} x_{1}^{\delta_{1}}+\cdots+\lambda_{n} x_{n}^{\delta_{n}}\right)}+\Psi_{\alpha}(x)
$$

form a finitely overcomplete set in $L^{p}\left(R_{+}^{n}\right)$. 
Alternatively, let $\gamma: \mathrm{R}^{n} \rightarrow \mathrm{C}$ be a function of polynomial growth such that $\gamma(x) \neq 0$ except possibly at 0 , and let $\left(\Psi_{\alpha}\right)_{\alpha \in \mathrm{N}^{n}}$ be a family of functions in $L^{p}\left(\mathrm{R}^{n}\right)$ such that $\lim \sup _{|\alpha| \rightarrow \infty}\left\|\Psi_{\alpha}\right\|_{p}^{1 /|\alpha|}=0$. Then for every $p$ with $1 \leq p<$ $\infty$, and for all $\lambda_{1}, \ldots, \lambda_{n}>0$ and $\delta_{1}, \ldots, \delta_{n} \geq 1$, the functions $f_{\alpha}$ defined by

$$
f_{\alpha}(x)=x^{\alpha} \gamma(x) e^{-\left(\lambda_{1}\left|x_{1}\right|^{\delta_{1}}+\cdots+\lambda_{n}\left|x_{n}\right|^{\delta_{n}}\right)}+\Psi_{\alpha}(x)
$$

form a finitely overcomplete set in $L^{p}(\mathrm{R})$.

Now let $\beta \geq-1$ and define the measure $\mu_{\beta}$ on $\mathbf{R}_{+}$by $d \mu_{\beta}(t)=d t / t^{\beta+1}$ for $t>0$. We then have the following result about overcompleteness in $L^{2}\left(\mathbf{R}_{+}, d \mu_{\beta}\right)$.

Corollary 3.6. Let $\Phi$ be a determining function on $\mathbf{R}_{+}$such that the function $x \mapsto \Phi(x)^{\epsilon}$ lies in $L^{2}\left(\mathbf{R}_{+}\right)$for some $\epsilon \in(0,1)$. Then the sequence of functions $\left(f_{m}\right)_{m}$ given by

$$
f_{m}(x)=x^{m} \Phi(x)+\Psi_{m}(x), \quad m \in \mathrm{N}, m>\beta / 2
$$

is finitely overcomplete in $L^{2}\left(\mathbf{R}_{+}, d \mu_{\beta}\right)$ whenever

$$
\lim _{m \rightarrow \infty}\left\|\Psi_{m}\right\|_{L^{2}\left(R_{+}, d \mu_{\beta}\right)}^{1 / m}=0
$$

Proof. This follows from Theorem 3.2 using the isometry

$$
J: L^{2}\left(\mathrm{R}_{+}\right) \rightarrow L^{2}\left(\mathrm{R}_{+}, d \mu_{\beta}\right)
$$

given by

$$
(J f)(x)=x^{(\beta+1) / 2} f(x),
$$

and noting that $x \mapsto x^{\delta} \Phi(x)$ is a determining function whenever $\delta>0$ and $\Phi$ is a determining function.

By means of a multiple of the Laplace transform, depending only on $\beta$, there is an isometric isomorphism between $L^{2}\left(\mathbf{R}_{+}, d \mu_{\beta}\right)$ for $\beta>-1$ and the weighted Bergman space $X_{\beta}=A_{\beta}^{2}\left(\mathrm{C}_{+}\right)$consisting of all analytic functions $F$ on the right half-plane $C_{+}$such that the norm

$$
\|F\|_{A_{\beta}^{2}\left(\mathrm{C}_{+}\right)}=\left(\frac{1}{\pi} \int_{\mathrm{C}_{+}}|F(x+i y)|^{2} x^{\beta} d x d y\right)^{1 / 2}
$$

is finite. The case corresponding to $\beta=-1$ (i.e. $d \mu_{\beta}(t)=d t$ ) is the Hardy space $X_{\beta}=H^{2}\left(\mathrm{C}_{+}\right)$where the norm is

$$
\|F\|_{H^{2}\left(\mathrm{C}_{+}\right)}=\left(\sup _{x>0} \int_{-\infty}^{\infty}|F(x+i y)|^{2} d y\right)^{1 / 2} .
$$


This Paley-Wiener type result can be found in [4], [5].

Under these isometric isomorphisms, the function $f$ given by $f(t)=$ $t^{\beta+1} e^{-\lambda t}$ with $\lambda>0$ corresponds to a multiple of the reproducing kernel $k_{\lambda}$ satisfying

$$
\left\langle F, k_{\lambda}\right\rangle_{X_{\beta}}=F(\lambda), \quad\left(F \in X_{\beta}\right) .
$$

Likewise $t \mapsto t^{m} f(t)$ corresponds to $(-1)^{m}$ times the $m$ th derivative of $k_{\lambda}$.

TheOREM 3.7. Let $\Phi$ be a determining function on $\mathbf{R}_{+}$such that $x \mapsto$ $\Phi(x)^{\epsilon} \in L^{2}\left(\mathbf{R}_{+}\right)$for some $\epsilon \in(0,1)$. For $\beta \geq-1$, define a function $k$ in $X_{\beta}$ by

$$
k(z)=\int_{0}^{\infty} x^{\beta+1} \Phi(x) e^{-x z} d x, \quad\left(z \in \mathrm{C}_{+}\right) .
$$

If $\left(G_{m}\right)_{m}$ is a sequence in $X_{\beta}$ with $\lim _{m \rightarrow \infty}\left\|G_{m}\right\|_{X_{\beta}}^{1 / m}=0$, then the functions $\left(k^{(m)}+G_{m}\right)_{m}$ form a finitely overcomplete set in $X_{\beta}$.

Proof. This follows from the properties of the Laplace transform as a mapping from $L^{2}\left(\mathrm{R}_{+}, d \mu_{\beta}\right)$ onto $X_{\beta}$ described above together with Corollary 3.6.

The analogue of Lin's result for $\mathrm{R}$, which is a special case of our Corollary 3.3, is that the functions $\left(x^{m} e^{-\lambda|x|}\right)_{m}$ are finitely overcomplete in $L^{2}(\mathbf{R})$. By using transform methods similar to those above, we obtain a further corollary of interest.

Corollary 3.8. Let $\Phi$ be a determining function on $\mathrm{R}$ such that $x \mapsto$ $\Phi(x)^{\epsilon} \in L^{2}(\mathrm{R})$ for some $\epsilon \in(0,1)$. Define a function $k$ in $L^{2}(\mathrm{R})$ by

$$
k(w)=\int_{-\infty}^{\infty} \Phi(x) e^{-i w x} d x, \quad(w \in \mathbf{R}) .
$$

If $\left(G_{m}\right)_{m}$ is a sequence in $L^{2}(\mathrm{R})$ with $\lim _{m \rightarrow \infty}\left\|G_{m}\right\|^{1 / m}=0$, then the functions $\left(k^{(m)}+G_{m}\right)_{m}$ form a finitely overcomplete set in $L^{2}(\mathbf{R})$.

Proof. Observe that $k$ is the Fourier transform of the function $x \rightarrow \Phi(x)$, and $i^{m} k^{(m)}$ is the transform of $x \mapsto x^{m} \Phi(x)$. The result now follows from the Plancherel theorem that the Fourier transform is a constant multiple of an isometry on $L^{2}(\mathrm{R})$.

Probably the most important application of Corollary 3.8 is obtained by taking $\Phi(x)=e^{-\lambda|x|}$ for $\lambda>0$, which gives

$$
k_{\lambda}(w)=\frac{\lambda}{\lambda^{2}+w^{2}},
$$

the Poisson kernel for the right half-plane (cf. [6, p. 123]). 


\section{Characterizing probability distributions}

In [10], [12], Lin and Too gave an application of the moment problem to the characterization of probability distributions via the moments of record values. In view of the results of this paper, it is possible to give more general versions of their results, and this is the aim of the present section.

Suppose $\left(X_{m}\right)_{m}$ be a sequence of independent, identically-distributed nonnegative random variables with continuous distribution $F$. Let $k$ be a positive integer and suppose that $E X_{1}^{p}<\infty$ for some $p>k$. The record times of the $X_{m}$, denoted $L(m)$, are defined by $L(0)=1$ and

$$
L(m)=\min \left\{k: X_{k}>X_{L(m-1)}\right\}, \quad(m \geq 1) .
$$

Then it follows that $E X_{L(m)}^{k}<\infty$ [11]. Let $\left(Y_{m}\right)_{m}$ be another such sequence, with continuous distribution $G$, and with corresponding record values $Y_{M(m)}$.

THEOREM 4.1. With the notation as above, suppose that $E X_{L(m)}^{k}=E Y_{M(m)}^{k}+$ $c_{m} / m$ !, and there is a number $c$ such that $\left|c_{m}-c\right|^{1 / m} \rightarrow 0$. Then $X$ and $Y$ have the same distribution.

Proof. We use a modification of the argument given in [12]. There it is shown that

$$
E X_{L(m)}^{k}=\frac{1}{m !} \int_{0}^{1}\left(F^{-1}(t)\right)^{k}\left(\log \frac{1}{1-t}\right)^{m} d t
$$

and we have

$$
\int_{0}^{1} f(t)\left(\log \frac{1}{1-t}\right)^{m} d t=\int_{0}^{1} g(t)\left(\log \frac{1}{1-t}\right)^{m}+c_{m}
$$

where $f(t)=\left(F^{-1}(t)\right)^{k}$ and $g(t)=\left(G^{-1}(t)\right)^{k}$. This becomes, by means of a change of variable $x=-\log (1-t)$,

$$
\int_{0}^{\infty} x^{m} h(x) d x=\int_{0}^{\infty} x^{m} l(x) d x+c_{m},
$$

with $h(x)=f(t) e^{-x}$ and $l(x)=g(t) e^{-x}$. The first term can be written

$$
\int_{0}^{\infty} x^{m} e^{-(p-1) x / p}\left(F^{-1}(t)\right)^{k} e^{-x / p} d x
$$

where $x \mapsto e^{-(p-1) x / p}$ is a determining function and $x \mapsto\left(F^{-1}(t)\right)^{k} e^{-x / p}$ lies in $L^{1}\left(R_{+}\right)$by Hölder's inequality. The end of the proof follows from Theorem 2.3. 
ACKNOWLEDGEMENTS. The authors are grateful to the EPSRC for financial support. They also wish to thank Dr. J. Stoyanov for drawing their attention to [12].

\section{REFERENCES}

1. Chalendar, I., Fricain, E., and Partington, J. R., Overcompleteness of sequences of reproducing kernels in model spaces, Integral Equations Operator Theory 56(1) (2006), 45-56.

2. Chalendar, I., Habsieger, L., Partington, J. R., and Ransford, T. J., Approximate Carleman theorems and a Denjoy-Carleman maximum principle, Arch. Math. (Basel) 83(1) (2004), 88-96.

3. Chalendar, I., and Partington, J. R., Multivariable approximate Carleman-type theorems for complex measures, Ann. Probab., to appear, 2006, Currently available at http://www. maths. leeds.ac.uk/ pmt6jrp/pubsjrp.html.

4. Das, N., and Partington, J. R., Little Hankel operators on the half-plane, Integral Equations Operator Theory 20(3) (1994), 306-324.

5. Duren, P., Gallardo-Gutiérrez, E. A., and Montes-Rodríguez, A., A Paley-Wiener theorem for Bergman spaces in the upper half-plane, Bull. London Math. Soc., to appear.

6. Hoffman, K., Banach Spaces of Analytic Functions, Dover Publications Inc., New York, 1988, Reprint of the 1962 original.

7. Kaiser, G., A friendly guide to wavelets, Birkhäuser Boston Inc., Boston, MA, 1994.

8. Koosis, P., The logarithmic integral I, Cambridge Stud. Adv. Math. 12.

9. Korenblum, B., Mascuilli, A., and Panariello, J., A generalization of Carleman's uniqueness theorem and a discrete Phragmén-Lindelöf theorem, Proc. Amer. Math. Soc. 126(7) (1998), 2025-2032.

10. Lin, G. D., On a moment problem, Tohoku Math. J. (2) 38(4) (1986), 595-598.

11. Lin, G. D., On characterizations of distributions via moments of record values, Probab. Theory Related Fields 74(4) (1987), 479-483.

12. Lin, G. D., and Too, Y. H., A moment problem and its application to characterization of distribution, Southeast Asian Bull. Math. 18(1) (1994), 85-88.

13. Singer, I., Bases in Banach Spaces. II, Springer-Verlag, Berlin-New York, 1981.

BÂT. JEAN BRACONMER

UNIVERSITÉ LYON 1, 43 BLD. DU 11/11/1918

69622 VILLEURBANNE CEDEX

FRANCE

E-mail: chalenda@math.univ-lyon1.fr
SCHOOL OF MATHEMATICS

UNIVERSITY OF LEEDS

LEEDS LS2 9JT

U.K.

E-mail: J.R.Partington@leeds.ac.uk 